TRANSACTIONS OF THE

AMERICAN MATHEMATICAL SOCIETY

Volume 348, Number 7, July 1996

\title{
ON FUNCTIONS IN THE LITTLE BLOCH SPACE AND INNER FUNCTIONS
}

\author{
S. ROHDE
}

\begin{abstract}
We prove that analytic functions in the little Bloch space assume every value as a radial limit on a set of Hausdorff dimension one, unless they have radial limits on a set of positive measure. The analogue for inner functions in the little Bloch space is also proven, and characterizations of various classes of Bloch functions in terms of their level sets are given.
\end{abstract}

\section{INTRODUCTION AND RESUlts}

In [R2] we considered the boundary behaviour of Bloch functions, i.e. functions $f$, analytic in the unit disk $\mathbb{D}$, for which

$$
\|f\|_{\mathcal{B}}=\sup _{z \in \mathbb{D}}\left(1-|z|^{2}\right)\left|f^{\prime}(z)\right|<\infty
$$

The space of all Bloch functions is denoted by $\mathcal{B}$. We introduced the class $\tilde{\mathcal{B}}$ of Bloch functions, normalized by $f(0)=0$ and $\|f\|_{\mathcal{B}}=1$, that have radial limits only on a set of zero measure, i.e. the set of points $\zeta \in \mathbb{T}$ for which the limit

$$
f(\zeta)=\lim _{r \rightarrow 1} f(r \zeta)
$$

exists is of (length) measure zero. An example of a function $f \in \tilde{\mathcal{B}}$ is $f(z)=$ $c \sum_{n \geq 1} z^{2^{n}}$ with a suitable $c$; see [P, Chapter 8].

In the present paper we mainly consider Bloch functions $f$ for which

$$
\lim _{r \rightarrow 1} \sup _{r<|z|<1}\left(1-|z|^{2}\right)\left|f^{\prime}(z)\right|=0 .
$$

These functions form the "little Bloch space", usually denoted by $\mathcal{B}_{0}$. See [A-C-P] for further information. Let

$$
\tilde{\mathcal{B}}_{0}=\mathcal{B}_{0} \cap \tilde{\mathcal{B}}
$$

be the class of normalized functions in $\mathcal{B}_{0}$ that have radial limits almost nowhere. An example is $f(z)=c \sum_{n \geq 1} \frac{1}{\sqrt{n}} z^{2^{n}}$ with appropriate $c$; see again $[\mathrm{P}]$. Our first result shows that the boundary behaviour of functions in $\tilde{\mathcal{B}}_{0}$ is rather pathological. For a set $E \subset \mathbb{T}$ we denote by $\operatorname{dim} E$ its Hausdorff dimension.

Received by the editors June 10, 1994.

1991 Mathematics Subject Classification. Primary 30D45.

Research performed in part as a Feodor Lynen Fellow of the Alexander von Humboldt-Stiftung. 
Theorem 1.1. Let $f \in \tilde{\mathcal{B}}_{0}$ and let $\gamma:[0,1) \rightarrow \mathbb{C}, \gamma(0)=0$, be an arbitrary continuous curve. Then there is a set $E_{\gamma} \subset \mathbb{T}$ with

$$
\operatorname{dim} E_{\gamma}=1
$$

so that for every $\zeta \in E_{\gamma}$ there is a homeomorphism $\phi_{\zeta}:[0,1) \rightarrow[0,1)$ such that

$$
\limsup _{r \rightarrow 1}\left|f(r \zeta)-\gamma\left(\phi_{\zeta}(r)\right)\right|=0 .
$$

Thus, given any function $f \in \tilde{\mathcal{B}}_{0}$ and any (continuous) curve $\gamma$ starting at 0 , there are "many" radii in $\mathbb{D}$ that are mapped onto curves "asymptotic" to the prescribed curve $\gamma$. A similar but necessarily weaker statement holds for functions in $\tilde{\mathcal{B}}$ and was proven in [R2]. Specializing Theorem 1.1 to line segments $\gamma$ from 0 to an arbitrary point $w \in \mathbb{C}$, we obtain

Corollary 1.2. For any $f \in \tilde{\mathcal{B}}_{0}$ and any $w \in \mathbb{C}$ the set

$$
E_{w}=\left\{\zeta \in \mathbb{T}: \lim _{r \rightarrow 1} f(r \zeta)=w\right\}
$$

has Hausdorff dimension 1.

Let $E$ denote the set of those points $\zeta \in \mathbb{T}$ where the function $f \in \tilde{\mathcal{B}}_{0}$ has finite radial limits. Carmona, Cufi and Pommerenke [C-C-P1] have shown that cap $E>0$, and Makarov [M2] improved this to $\operatorname{dim} E=1$. This follows also from Corollary 1.2 , since $E_{w} \subset E$ for all $w$.

Remark. The assumption $f \in \tilde{\mathcal{B}}$ in Theorem 1.1 and Corollary 1.2 can be weakened to the following: there is an arc $A \subset \mathbb{T}$ such that $f$ has radial limits almost nowhere on $A$. The proof of this requires only minor technical changes. After Lemma 2.3 and the proof of Theorem 1.1 below we comment on these changes and leave the details to the reader.

An interesting consequence of Corollary 1.2 (under the weakened hypotheses of the remark) is the following purely geometric result:

Corollary 1.3. Let $C$ be an asymptotically conformal Jordan curve that has tangents on a set of linear measure zero only. Then, for every direction $t \in[0,2 \pi]$ there is a subset $C_{t} \subset C$ of dimension 1 so that $C$ has a tangent of direction $t$ at every point $w \in C_{t}$.

A function $f$, analytic in the unit disk, is an inner function if $|f(z)|<1$ in $\mathbb{D}$ and if the radial limits $f(\zeta)$ satisfy $|f(\zeta)|=1$ for almost every $\zeta \in \mathbb{T}$. The singular set $S(f)$ of an inner function $f$ is the set of accumulation points of the zeros of $f$, together with the support of the singular measure on $\mathbb{T}$ appearing in the canonical factorization [G, Chapter 2.5].

Theorem 1.4. Let $f \in \mathcal{B}_{0}$ be an inner function which is not a finite Blaschke product. For $y \in \mathbb{D}$, consider

$$
E_{y}=\{\zeta \in \mathbb{T}: f(\zeta)=y\}
$$

Then $\operatorname{dim} E_{y}=1$.

See $[B]$ for a characterization of inner functions in $\mathcal{B}_{0}$. Hungerford $[\mathrm{H}]$ has established a conjecture of Wolff, proving that the singular set of inner functions in $\mathcal{B}_{0}$ 
has dimension 1. Since $E_{y} \subset S(f)$ for any $y \in \mathbb{D}$, Theorem 1.4 is an improvement of this result. Furthermore, Corollary 1.2 is a simple consequence of Theorem 1.4; see section 3 .

In section 2 we will discuss level sets of Bloch functions and modify some results from [R2] to functions in $\mathcal{B}_{0}$.

Theorems 1.1, 1.4 and Corollary 1.3 are proven in section 3.

Section 4 is independent from section 3 . There we will give various characterizations of subclasses of $\mathcal{B}$ in terms of level sets (Theorem 4.1), in the spirit of the work of Stegenga and Stephenson [S-S].

Acknowledgement. Some of the results of this paper were already contained in my doctoral thesis at the Technische Universität Berlin. I would like to thank my adviser Christian Pommerenke for many stimulating discussions.

\section{Level Sets}

Let $f$ be a Bloch function with $\|f\|_{\mathcal{B}}=1, z_{0} \in \mathbb{D}, a>0$ and consider the component (level set) $\Omega_{a}=\Omega_{a}\left(f, z_{0}\right)$ of $\left\{z \in \mathbb{D}:\left|f(z)-f\left(z_{0}\right)\right|<a\right\}$ containing $z_{0}$. It is known ([S-S], [GH-P]; see also [R2]) that $\bar{\Omega}_{a} \cap \mathbb{T} \neq \emptyset$ if $a>e / 2$. In the case $\Omega_{a} \neq \mathbb{D}$, set

$$
\mathbb{T} \backslash \overline{\Omega_{a}\left(f, z_{0}\right)}=\bigcup_{n} I_{n}
$$

with disjoint open $\operatorname{arcs} I_{n}=I_{n}\left(f, z_{0}, a\right)$. If $a>e \pi / 2$, then $\left|I_{n}(f, 0, a)\right|<1 / 2$ (and thus the same is true for $z_{0} \neq 0$ if we replace length by harmonic measure). This easily follows from [P, Theorem 4.2] and is contained in the proof of Lemma 2.1 in [R2]. We modify the simply connected domain $\Omega_{a}$ to a new simply connected domain $G_{a}=G_{a}\left(f, z_{0}\right)$ by replacing the components of $\partial \Omega_{a} \cap \mathbb{D}$ by circular arcs that intersect $\mathbb{T}$ in a fixed angle. To be more precise, let $B_{n}$ be the circular arc in $\overline{\mathbb{D}}$ that intersects $\mathbb{T}$ at the endpoints of $I_{n}$ under the angle $\beta$, where $0<\beta<\pi / 2$ is fixed. Let $G_{a}$ be the component of $\mathbb{D} \backslash \bigcup B_{n}$ containing $z_{0}$. We will use the notation $z_{n}, z\left(B_{n}\right), z\left(I_{n}\right)$ for the midpoint of $B_{n}$.

The following statement is [R2, Lemma 2.1] (there we made the assumption $f \in \tilde{\mathcal{B}}$, but the proof does not need this).

Lemma 2.1. Let $f \in \mathcal{B},\|f\|_{\mathcal{B}} \leq 1, a>\frac{e \pi}{2}$ and $G_{a}=G_{a}(f, 0)$. There are constants $K_{1}$ and $K_{2}$, depending only on $\beta$, so that for the arcs $I_{n}$ and $B_{n}$ described above we have

$$
\begin{gathered}
a-K_{1} \leq|f(z)| \leq a+K_{1} \quad \text { for } z \in B_{n}, \\
|f(z)| \leq a+K_{1} \quad \text { in } G_{a}(f, 0)
\end{gathered}
$$

and

$$
\left|I_{n}\right| \leq K_{2} e^{-a} .
$$

Thus $\Omega_{a-K_{1}} \subset G_{a} \subset \Omega_{a+K_{1}}$ and one could think of the domains $G_{a}$ themselves as level sets of $f$.

The next lemma is a modification of [R2, Theorem 2.2]. The only difference is that we relax the condition $f \in \tilde{\mathcal{B}}$.

For points $w \in \mathbb{C} \backslash\{0\}$ denote by $p(w)$ the projection of $w$ onto $\mathbb{T}$,

$$
p(w)=\frac{w}{|w|} .
$$


Lemma 2.2. Let $f \in \mathcal{B}, f(0)=0,\|f\|_{\mathcal{B}} \leq 1, a>e \pi / 2$, and consider the domain $G_{a}=G_{a}(f, 0)$ (together with the arcs $I_{n} \subset \mathbb{T}$ and points $z_{n}$ as defined above). Let $A \subset \mathbb{T}$ be an arc and let $E \subset \mathbb{T}$ be another arc with

$$
\left|\left\{\zeta \in \mathbb{T}: \limsup _{r \rightarrow 1}|f(r \zeta)| \leq a+K_{1}\right\} \backslash E\right|=0 .
$$

Set

$$
J=\left\{j: p\left(f\left(z_{j}\right)\right) \in A \text { and } I_{j} \cap E=\emptyset\right\} .
$$

Then

$$
\sum_{j \in J}\left|I_{j}\right| \geq\left(1-\frac{2 \beta}{\pi}\right)|A|-c(|E|)
$$

for $a \geq a_{0}(|A|)$, where $c(t) \rightarrow 0$ as $t \rightarrow 0$. The constants $a_{0}($.$) and c($.$) do not$ depend on $f$.

Proof. As in the proof of Lemma 2.3 in [R2] we consider the conformal map $\phi$ : $\mathbb{D} \rightarrow G_{a}, \phi(0)=0$, and set

$$
h(z)=\frac{1}{a+K_{1}} f(\phi(z)) .
$$

Then $|h(z)| \leq 1$ by (2.3). The assumptions on $f$ and $E$ imply that $\left|\partial G_{a} \cap(\mathbb{T} \backslash E)\right|=$ 0 , so that by $(2.2)$

$$
|h(\zeta)| \geq \frac{a-K_{1}}{a+K_{1}}
$$

for almost every $\zeta \in \mathbb{T} \backslash \phi^{-1}(E)$. The proof of Lemma 2.3 in [R2] shows that

$$
\left|\left\{\zeta \in \mathbb{T} \backslash \phi^{-1}(E): p(h(\zeta)) \in A\right\}\right| \geq|A|-\left|\phi^{-1}(E) \cap \mathbb{T}\right|-o(1)
$$

as $a \rightarrow \infty$, where the $o(1)$ does not depend on $f$. Observing that $\left|\phi^{-1}(E) \cap \mathbb{T}\right| \leq$ $c(|E|)$, the rest of the proof is as the proof of Theorem 2.2 in [R2].

The next lemma is crucial in the proofs of Theorems 1.1 and 1.4. For a function $f \in \mathcal{B}_{0}$ we set

$$
\mu(r)=\mu_{f}(r)=\sup _{r<|z|<1}\left(1-|z|^{2}\right)\left|f^{\prime}(z)\right| .
$$

For a real number $0<r<1$ we denote by $\rho(r)$ the midpoint of the arc $[0, r] \subset \mathbb{D}$ in the hyperbolic metric; hence $0<\rho(r)<r$ and $\rho(r) \rightarrow 1$ as $r \rightarrow 1$. Given an angle $0<\beta \leq \pi / 2$ and a point $z \in \mathbb{D} \backslash\{0\}$, there is a unique arc $I \subset \mathbb{T}$ so that $z$ is the midpoint of the circular arc $B$ through the endpoints of $I$, intersecting $\mathbb{T}$ in the angle $\beta$. We denote this arc by $I(z)$. For points $z, w \in \mathbb{D}$ we denote by $\langle z, w\rangle$ the noneuclidean line segment between $z$ and $w$.

Lemma 2.3. Let $f \in \tilde{\mathcal{B}}_{0}, \zeta_{0} \in \mathbb{D}, w \in \mathbb{C}$, or let $f \in \mathcal{B}_{0}$ be an inner function, $\zeta_{0} \in \mathbb{D}$ and $w \in \mathbb{D}$. Set

$$
\alpha=\left|f\left(\zeta_{0}\right)-w\right|
$$

and assume that

$$
\left|f\left(\zeta_{0}\right)\right|<1-\alpha-K_{1} \mu\left(\rho\left(\left|\zeta_{0}\right|\right)\right)
$$


if $f$ is inner (if $f \in \tilde{\mathcal{B}}_{0}$ we need no further assumption). Then there are universal constants $0<c<1, C>1$ and $\beta$ so that the following holds.

If $\alpha>C \mu\left(\rho\left(\left|\zeta_{0}\right|\right)\right)$ and $\left|\zeta_{0}\right|>1-c$, then there are points $\zeta_{j} \in \mathbb{D}$ such that

$$
\left|f\left(\zeta_{j}\right)-w\right| \leq \frac{\alpha}{2}
$$

$$
I\left(\zeta_{j}\right) \subset I\left(\zeta_{0}\right) \text { and the } I\left(\zeta_{j}\right), j \geq 1 \text {, are pairwise disjoint, }
$$

$$
\begin{gathered}
\left|\bigcup_{j} I\left(\zeta_{j}\right)\right| \geq c\left|I\left(\zeta_{0}\right)\right| \\
\left|f(z)-f\left(z^{\prime}\right)\right| \leq C \alpha \quad \text { for } \quad z, z^{\prime} \in\left\langle\zeta_{0}, \zeta_{j}\right\rangle \\
\left|I\left(\zeta_{j}\right)\right| \leq C \exp \left(-\frac{1}{C} \frac{\alpha}{\mu\left(\rho\left(\left|\zeta_{0}\right|\right)\right)}\right)\left|I\left(\zeta_{0}\right)\right| .
\end{gathered}
$$

Proof. Consider the Möbius transformation

$$
T(z)=\frac{z-\zeta_{0}}{1-\overline{\zeta_{0}} z}
$$

Let $\zeta_{0}^{\prime}=\rho\left(\left|\zeta_{0}\right|\right) \zeta_{0}$ be the midpoint of the radius $\left[0, \zeta_{0}\right]$ in the hyperbolic metric and let $D$ be the disk $D=\left\{|z| \leq\left|\zeta_{0}^{\prime}\right|\right\}$. Then $T(D)$ is a disk of hyperbolic distance $\rho\left(\left|\zeta_{0}\right|\right)$ to 0 . Thus there is a point $\xi \in \mathbb{T}$ and a domain $H_{\delta} \subset \mathbb{D}$ of the form $H_{\delta}=\{z \in \mathbb{D}:|z-\xi|>\delta\}$ so that $T(D) \subset \mathbb{D} \backslash H_{\delta}$ (we could choose $\left.\xi=T\left(\zeta_{0}^{\prime}\right) /\left|T\left(\zeta_{0}^{\prime}\right)\right|\right)$. Furthermore $\delta \rightarrow 0$ as $\left|\zeta_{0}\right| \rightarrow 1$. Let $\phi: \mathbb{D} \rightarrow H_{\delta}$ be the conformal map, normalized by $\phi(0)=0, \phi^{\prime}(0)>0$. Reflection and a normal families argument shows that

$$
\left|\left(\phi^{-1}\right)^{\prime}(\zeta)-1\right|<\varepsilon
$$

for $|\zeta-\xi|>\varepsilon$, where $\varepsilon \rightarrow 0$ as $\delta \rightarrow 0$.

Now consider the function

$$
g(z)=f\left(T^{-1}(\phi(z))\right)
$$

If we write $x=T^{-1}(\phi(z))$, the chain rule gives

$$
\left(1-|z|^{2}\right)\left|g^{\prime}(z)\right| \leq\left(1-|z|^{2}\right)\left|f^{\prime}(x)\right| \frac{1-|x|^{2}}{1-|\phi(z)|^{2}}\left|\phi^{\prime}(z)\right| \leq\left(1-|x|^{2}\right)\left|f^{\prime}(x)\right| \leq \mu\left(\rho\left(\left|\zeta_{0}\right|\right)\right) .
$$

The last inequality follows from the definition of $\mu$ and the fact that $|x| \geq \rho\left(\left|\zeta_{0}\right|\right)$ since $\phi$ maps into $H_{\delta}$.

We have thus shown that $h(z)=(g(z)-g(0)) / \mu\left(\rho\left(\left|\zeta_{0}\right|\right)\right)$ has $\|h\|_{\mathcal{B}} \leq 1$. Let

$$
E=\{\zeta \in \mathbb{T}:|\phi(\zeta)-\xi|<\varepsilon\}
$$


and

$$
a=\alpha / \mu\left(\rho\left(\left|\zeta_{0}\right|\right)\right) .
$$

If $f \in \tilde{\mathcal{B}}_{0}$ then $\lim \sup _{r \rightarrow 1}|h(r \zeta)|=\infty$ for almost every $\zeta \notin E$, whereas

$$
\limsup _{r \rightarrow 1}|h(r \zeta)| \geq\left(1-\left|f\left(\zeta_{0}\right)\right|\right) / \mu\left(\rho\left(\left|\zeta_{0}\right|\right)\right) \geq a+K_{1}
$$

for a.e. $\zeta \notin E$ if $f$ is inner. In both cases, we can apply Lemma 2.2 to $w^{\prime}=$ $\left(w-f\left(\zeta_{0}\right)\right) / \mu\left(\rho\left(\zeta_{0}\right)\right), A=p\left(\left\{z:\left|z-w^{\prime}\right| \leq a / 4\right\}\right)$ and the above $h, E$ and $a$. Assuming that $1-\left|\zeta_{0}\right|$ is small enough, it follows that the right hand side of (2.5) is positive. We obtain points $z_{j}$ and a set $J$ of indices. Then $\left|h\left(z_{j}\right)-w^{\prime}\right| \leq a / 2$, and setting $\zeta_{j}=T^{-1}\left(\phi\left(z_{j}\right)\right)$ for $j \in J$, (I) follows at once. Furthermore (II), (III) and (V) follow easily from Lemma 2.2 and (2.7), if the angle $\beta$ in Lemma 2.3 is chosen somewhat larger than the $\beta$ in Lemma 2.2. Finally, (IV) follows from Pommerenke's estimate [P, Theorem 4.2].

Remark. If $f \in \mathcal{B}_{0}, I \subset \mathbb{T}$ is an arc, and $f$ has finite radial limits almost nowhere on $I$, then the proof of Lemma 2.3 still works, provided $\zeta_{0}$ is near $I$ (i.e. the harmonic measure of $I$ at $\zeta_{0}$ is close to one). This is clear because (in the notation above) we can choose $\varepsilon$ so that $E \supset \phi^{-1}(T(I))$.

\section{Proofs}

As in [R2] we will use the following lower bound for Hausdorff dimension due to Hungerford [H] and Makarov [M2]; see also [P, Chapter 10].

Lemma 3.1. Let $a>0$ and $0<b<1$. Let $I_{n}^{(k)}(n, k=0,1,2, \ldots)$ be a family of arcs on $\mathbb{T}$ that are pairwise disjoint for fixed $k$, so that for every $I_{n}^{(k)}$ there is an $I_{m}^{(k-1)} \supset I_{n}^{(k)}$ with

$$
\left|I_{n}^{(k)}\right| \leq e^{-a}\left|I_{m}^{(k-1)}\right|
$$

and furthermore

$$
\sum_{I_{m}^{(k+1)} \subset I_{n}^{(k)}}\left|I_{m}^{(k+1)}\right| \geq b\left|I_{n}^{(k)}\right|
$$

for all $n, k \geq 0$. Then

$$
\operatorname{dim} \bigcap_{k \geq 0} \bigcup_{n} I_{n}^{(k)} \geq 1-\frac{\log (1 / b)}{a}
$$

Proof of Theorem 1.1. Choose a sequence $\gamma_{n}=\gamma\left(t_{n}\right)$ of points on $\gamma$ such that $t_{n}$ increases to 1 and

$$
\max _{t_{n} \leq t \leq t_{n+1}}\left|\gamma(t)-\gamma_{n}\right|
$$

decreases to zero. We may assume that $\gamma_{n+1} \neq \gamma_{n}$ for all $n$, otherwise replace $\gamma_{n}$ by $\gamma_{n}+\varepsilon_{n}$ for some sequence $\varepsilon_{n} \rightarrow 0$. 
Similar to the procedure in [R2], we construct $\operatorname{arcs} I_{n}^{(k)}$ having certain properties (a)-(d) below. The points $z_{n}^{(k)}=z\left(I_{n}^{(k)}\right.$ ) (we fix the angle $\beta$ of Lemma 2.3) can be thought of as vertices of a tree in $\mathbb{D}$, where $z_{n}^{(k)}$ and $z_{m}^{(k+1)}$ are joined by a geodesic $\operatorname{arc}\left\langle z_{n}^{(k)}, z_{m}^{(k+1)}\right\rangle$ if $I_{m}^{(k+1)} \subset I_{n}^{(k)}$. By a branch we mean a sequence $z_{n_{k}}^{(k)}$ of these points with the property $I_{n_{k+1}}^{(k+1)} \subset I_{n_{k}}^{(k)}$.

The construction will be made so that

(a) there is a sequence $a_{k} \rightarrow \infty$ such that (3.1) holds with $a$ replaced by $a_{k}$,

(b) for some universal constant $b,(3.2)$ holds for all $n$ and $k$,

(c) there is a sequence $\delta_{k} \rightarrow 0$ such that

$$
\left|f\left(z_{n}^{(k)}\right)-\gamma_{k}\right| \leq \delta_{k}
$$

for all $n$ and $k$,

(d) there is a sequence $\varepsilon_{k} \rightarrow 0$ such that $\left|f(z)-f\left(z^{\prime}\right)\right| \leq \varepsilon_{k}$ on geodesics $\left\langle z_{n}^{(k)}, z_{m}^{(k+1)}\right\rangle$ if $I_{m}^{(k+1)} \subset I_{n}^{(k)}$.

We will then show that $\bigcap_{k>0} \bigcup_{n} I_{n}^{(k)}$ has the desired properties.

We start the inductive construction with some $\operatorname{arc} I_{0}^{(0)} \subset \mathbb{T}$ for which $\left|z_{0}^{(0)}\right|>1-c$ and $\mu=\mu\left(\rho\left(\left|z_{0}^{(0)}\right|\right)\right)$ is so small that

$$
C \exp \left(-\frac{1}{C \sqrt{\mu}}\right)<\frac{1}{2}
$$

where $c$ and $C$ are as in Lemma 2.3.

Having constructed all points $z_{n}^{(\kappa)}$ for $\kappa \leq k$, fix a point $\zeta_{0}=z_{n}^{(k)}$, and set $\mu=\mu\left(\rho\left(\left|\zeta_{0}\right|\right)\right)$ and

$$
\alpha=\max \left\{\left|f\left(\zeta_{0}\right)-\gamma_{k+1}\right|, \sqrt{\mu}\right\} .
$$

If $\alpha=\sqrt{\mu}$ set $w=f\left(\zeta_{0}\right)+\sqrt{\mu}$, otherwise set $w=\gamma_{k+1}$. In any case we have $\left|f\left(\zeta_{0}\right)-w\right|=\alpha$. An application of Lemma 2.3 yields points $\zeta_{j}=\zeta_{j}\left(z_{n}^{(k)}\right) \in \mathbb{D}$ with various properties. We define the points $z_{m}^{(k+1)}(m=1,2,3, \ldots)$ to be an enumeration of the points $\zeta_{j}\left(z_{n}^{(k)}\right)(n, j=1,2,3, \ldots)$. This just means that the $(k+1)$-st level of the tree is given by the "children" of the points of level $k$ in the tree.

This finishes the construction.

To verify (a)-(d), note that our construction implies

$$
\left|z_{n}^{(k)}\right| \rightarrow 1
$$

as $k \rightarrow \infty$, uniformly in $n$. In fact, (3.5) and (V) of Lemma 2.3 imply $1-\left|z_{m}^{(k+1)}\right| \leq$ $\frac{1}{2}\left(1-\left|z_{n}^{(k)}\right|\right)$. Set $\mu_{k}=\sup _{n}\left\{\mu\left(\rho\left(\left|z_{n}^{(k)}\right|\right)\right)\right\}$; it follows that $\mu_{k} \rightarrow 0$ as $k \rightarrow \infty$. Thus (a) follows from (V) and (b) follows from (III) in Lemma 2.3.

To see (c), again fix $\zeta_{0}=z_{n}^{(k)}$ and consider one of its children $z_{n_{j}}^{(k+1)}$.

If $\alpha=\sqrt{\mu}$ then

$$
\left|f\left(z_{n_{j}}^{(k+1)}\right)-\gamma_{k+1}\right|=\left|f\left(z_{n_{j}}^{(k+1)}\right)-\left(f\left(\zeta_{0}\right)+\sqrt{\mu}\right)+\left(f\left(\zeta_{0}\right)-\gamma_{k+1}\right)+\sqrt{\mu}\right| \leq \frac{5}{2} \sqrt{\mu}
$$


otherwise

$$
\left|f\left(z_{n_{j}}^{(k+1)}\right)-\gamma_{k+1}\right| \leq \frac{1}{2}\left|f\left(\zeta_{0}\right)-\gamma_{k+1}\right| \leq \frac{1}{2}\left|f\left(\zeta_{0}\right)-\gamma_{k}\right|+\frac{1}{2}\left|\gamma_{k}-\gamma_{k+1}\right| .
$$

We see that the inductively defined sequence

$$
\delta_{k+1}=\frac{1}{2} \delta_{k}+\frac{1}{2}\left|\gamma_{k}-\gamma_{k+1}\right|+\frac{5}{2} \sqrt{\mu_{k}}
$$

satisfies (c).

Finally, (d) follows from (3.6), (IV) of Lemma 2.3 and (c), if we set $\varepsilon_{k}=$ $C\left(\delta_{k}+\left|\gamma_{k}-\gamma_{k+1}\right|+\sqrt{\mu_{k}}\right)$. Set

$$
E=\bigcap_{k \geq k_{0}} \bigcup_{n} I_{n}^{(k)}
$$

As we can choose $k_{0}$ arbitrarily large, (a), (b) and Lemma 3.1 show that

$$
\operatorname{dim} E=1 .
$$

We want to show that $E_{\gamma}=E$ satisfies (1.2). Take a point $\zeta \in E$. Then there is a branch $z_{n_{k}}^{(k)}$ such that $\bigcap_{k} I_{n_{k}}^{(k)}=\{\zeta\}$. The geodesic polygonal arc $\bigcup_{k}\left\langle z_{n_{k}}^{(k)}, z_{n_{k+1}}^{(k+1)}\right\rangle$ lies within a bounded hyperbolic distance of the radius $[0, \zeta$ ) (the bound depends only on $\beta$, which is fixed). Parametrize this polygon by $\sigma(t), t_{0} \leq t<1$, so that $|\sigma(t)|=t$. Then $|f(t \zeta)-f(\sigma(t))| \rightarrow 0$ as $t \rightarrow 1$ since $f \in \mathcal{B}_{0}$. On the other hand, (c) and (d) imply that $\gamma$ can be reparametrized (call this $\tilde{\gamma}$ ) so that $|f(\sigma(t))-\tilde{\gamma}(t)| \rightarrow 0$ as $t \rightarrow 1$. Thus (1.2) holds and the theorem is proven.

Remark. The above proof works as soon as we can apply Lemma 2.3. Together with the remark after Lemma 2.3 we thus obtain the slightly stronger version of Theorem 1.1, mentioned in the remark after Theorem 1.1.

Proof of Corollary 1.3. Let $\phi: \mathbb{D} \rightarrow \operatorname{Int}(C)$ be conformal and set $f=\log \phi^{\prime}$. As $C$ is asymptotically conformal, $f \in \mathcal{B}_{0}$. It is standard that the set $R \subset \mathbb{T}$, where $f$ has finite radial limits is of measure zero: The angular derivative of $\phi$ exists on $R$ so that $C$ has tangents at $\phi(\zeta)$ for all $\zeta \in R$ by [P, Theorem 5.5]. By the McMillan sector theorem (see $[\mathrm{P}$, Theorem 6.24]) $|R|=0$.

Let $e^{i x} \in R$. Then the direction (angle) $t$ of the tangent at $\phi\left(e^{i x}\right)$ is $t=$ $\arg \phi^{\prime}\left(e^{i x}\right)+x+\pi / 2=\operatorname{Im}\left(f\left(e^{i x}\right)\right)+x+\pi / 2$. We would like to apply Corollary 1.2 to $f(z)+\log z$. As this function is not analytic in $\mathbb{D}$, we consider the domain $D=\{z \in \mathbb{D}: \operatorname{Im}(\mathrm{z})>1 / 2\}$, a conformal map $\psi: \mathbb{D} \rightarrow D$ and the function $g(z)=f(\psi(z))+\log (\psi(z))+i \pi / 2$, which is analytic in $\mathbb{D}$. The chain rule shows that $g \in \mathcal{B}_{0}$, and it is clear that $g$ has almost no radial limits on the arc $A=\psi^{-1}(\mathbb{T})$.

Fix $t \in[0,2 \pi]$. By the remark after Corollary 1.2 there is a set $E \subset A$ so that $g$ has the radial limit $g(\zeta)=i t$ for all $\zeta \in E$, and $\operatorname{dim} E=1$. Therefore $f\left(e^{i x}\right)+i x+i \pi / 2=i t$ at $e^{i x}=\psi(\zeta)$ and it follows that $C$ has a tangent of direction $t$ at $\phi(\psi(\zeta))$. It remains to note that $\operatorname{dim} \phi(\psi(E)) \geq 1$ (and hence $=1$ since $C$ is asymptotically conformal) by a result of Makarov [M1], [P, Chapter 10].

Proof of Theorem 1.4. We may assume that $f$ is an infinite Blaschke product and that $|y| \leq 1 / 8$. To see this, consider the automorphisms $T_{\alpha}(z)=(z-\alpha) /(1-\bar{\alpha} z)$ 
of $\mathbb{D}$ and the inner functions $f_{\alpha}=T_{\alpha} \circ f \in \mathcal{B}_{0}$. By a theorem of Frostman (see [G, Theorem 6.4]), $f_{\alpha}$ is a Blaschke product for a dense set of values of $\alpha$. Choose $\alpha$ so that $\left|T_{\alpha}(y)\right| \leq 1 / 8$.

As in the proof of Theorem 1.1, we will construct $\operatorname{arcs} I_{n}^{(k)}$ (or rather the corresponding points $\left.z_{n}^{(k)}\right)$, having the properties (a), (b) and (d) above and instead of (c)

(c') there is a sequence $\delta_{k} \rightarrow 0$ as $k \rightarrow \infty$ such that $\left|f\left(z_{n}^{(k)}\right)-y\right| \leq \delta_{k}$.

These properties easily imply Theorem 1.4 (see the proof of Theorem 1.1).

Fix a number

$$
\mu_{0} \leq \max \left(\frac{1}{4 K_{1}}, \frac{1}{400}, \frac{1}{C^{2}}\right)
$$

such that

$$
C \exp \left(-\frac{1}{C \sqrt{\mu_{0}}}\right)<\frac{1}{2}
$$

where $K_{1}$ and $C$ are from Lemma 2.3. The inductive construction will be made so that the $z_{n}^{(k)}$ have the additional property

$$
\mu\left(\rho\left(\left|z_{n}^{(k)}\right|\right)\right)<\mu_{0} \quad \text { and } \quad\left|f\left(z_{n}^{(k)}\right)\right|<\frac{1}{4} .
$$

We start the construction with some point $z_{0}^{(0)}$ for which $f\left(z_{0}^{(0)}\right)=0$ and $\mu\left(\rho\left(\left|z_{0}^{(0)}\right|\right)\right)<\mu_{0}$. This is the only place where we take advantage of the assumption that $f$ is not a finite Blaschke product. Then (c') is satified for $k=0$ with $\delta_{0}=1 / 8$.

Given a point $\zeta_{0}=z_{n}^{(k)}$, we construct it's "children" $\zeta_{j}=z_{n_{j}}^{(k+1)}$ as follows: Set $\mu=\mu\left(\rho\left(\left|\zeta_{0}\right|\right)\right)$ and

$$
\alpha=\max \left\{\left|f\left(\zeta_{0}\right)-y\right|, \sqrt{\mu}\right\} .
$$

If $\alpha=\sqrt{\mu}$ set $w=f\left(\zeta_{0}\right)+\sqrt{\mu}$; otherwise set $w=y$. By the induction hypothesis (3.8), the condition (2.6) is satisfied. As $\mu<\mu_{0} \leq 1 / C^{2}$ we have $\alpha>C \mu$, so that an application of Lemma 2.3 yields the desired points $\zeta_{j}$.

Note that $\left|\zeta_{j}\right|>\left|\zeta_{0}\right|$ by (II) in Lemma 2.3, so that $\mu\left(\rho\left(\left|\zeta_{j}\right|\right)\right)<\mu_{0}$, i.e. the first inequality of (3.8) holds for $k+1$. By (I) in Lemma 2.3, if $\alpha=\sqrt{\mu}$ we have

$$
\left|f\left(\zeta_{j}\right)-y\right| \leq\left|f\left(\zeta_{j}\right)-f\left(\zeta_{0}\right)\right|+\left|f\left(\zeta_{0}\right)-y\right| \leq \frac{5}{2} \sqrt{\mu},
$$

whereas

$$
\left|f\left(\zeta_{j}\right)-y\right| \leq \frac{1}{2}\left|f\left(\zeta_{0}\right)-y\right|
$$

otherwise. In both cases, using (3.8) and $\mu_{0} \leq 1 / 400$ we see that $\left|f\left(\zeta_{j}\right)\right|<1 / 4$ and have thus verified (3.8) for $k+1$.

Again we see from (V) in Lemma 2.3 and the assumption on $\mu_{0}$ that

$$
\left|z_{n}^{(k)}\right| \rightarrow 1
$$

as $k \rightarrow \infty$, uniformly in $n$, so that (a), (b) and (d) follow from (II), (III) and (IV) in Lemma 2.3. With $\mu_{k}=\sup _{n}\left\{\mu\left(\rho\left(\left|z_{n}^{(k)}\right|\right)\right)\right\}$, the inductively defined sequence

$$
\delta_{k+1}=\frac{\delta_{k}}{2}+\frac{5}{2} \sqrt{\mu_{k}}
$$

fulfills (c') by (3.9) and (3.10). The proof is finished. 
Remark. Corollary 1.2 also follows from Theorem 1.4: In [C-C-P2] inner functions in $\mathcal{B}_{0}$ were constructed by composing functions $f \in \tilde{\mathcal{B}}_{0}$ with a conformal map $\phi: \mathbb{D} \rightarrow \Omega_{a}(f, 0)$ (that is, $h=\frac{1}{a} f \circ \phi$ is inner and in $\left.\mathcal{B}_{0}\right)$. If $a$ is large enough, $h$ will not be a finite Blaschke product. Now Theorem 1.4 implies that $f \circ \phi$ assumes every $w \in \mathbb{C}$ with $|w|<a$ as a radial limit on a set of dimension 1. Since $\phi$ preserves dimension 1 (on $\mathbb{T}$ ), the corollary follows.

\section{Characterizations of Bloch functions}

We now turn to characterizations of $\tilde{\mathcal{B}}, \mathcal{B}_{0}$, BMOA, VMOA and a further subclass of $\mathcal{B}$ in terms of the level sets. We need some notation.

For $f \in \mathcal{B}, z \in \mathbb{D}$ and $a>0$ let

$$
\omega_{a}(z)=\omega_{\mathbb{D}}\left(z, \overline{\Omega_{a}(f, z)} \cap \mathbb{T}\right)
$$

be the harmonic measure in $\mathbb{D}$ of $\mathbb{T} \backslash \bigcup I_{n}(f, z, a)$ (see (2.1)). This is similar to the quantity $\omega_{a}(r)$ of $[\mathrm{S}-\mathrm{S}]$, but there harmonic measure was with respect to $\Omega_{a}$ instead of $\mathbb{D}$. (Also our notation differs from $[\mathrm{S}-\mathrm{S}]$ in that our $a$ corresponds to their $r$, whereas our $z$ corresponds to their $a$.) We will use the notation $\tilde{\omega}_{a}(z)=\omega_{\Omega_{a}}(z, \mathbb{T})$; hence $\omega_{a} \geq \tilde{\omega}_{a}$ by the maximum principle. Let

$$
l_{a}(z)=\max _{n} \omega_{\mathbb{D}}\left(z, I_{n}\right)
$$

be the "size" of the largest component $I_{n}=I_{n}(f, z, a)$. We set $l_{a}(z)=1$ if $\overline{\Omega_{a}(z)} \subset$ $\mathbb{D}$, and $l_{a}(z)=0$ if $\Omega_{a}=\mathbb{D}$. Thus

$$
l_{a}(z) \leq 1-\omega_{a}(z)
$$

The classes $\tilde{\mathcal{B}}$ and $\mathcal{B}_{0}$ were mentioned in the first chapter. BMOA and VMOA are defined as usual, see [G, Chapter VI] or [P, Chapter 7]. We denote the BMO-norm of $f$ by $\|f\|_{*}$.

Additionally we consider the class $\mathcal{J}$ of Bloch functions $f$ with the following property: There is a constant $\varepsilon>0$ so that in every disk $D \subset \mathbb{D}$ of hyperbolic radius 1 there is a point $z$ with

$$
\left(1-|z|^{2}\right)\left|f^{\prime}(z)\right| \geq \varepsilon .
$$

These functions have been considered by Jones [J]. Let $g$ be a conformal mapping of $\mathbb{D}$ onto a quasidisk $G$ and set $f=\log g^{\prime}$. Then $f \in \mathcal{J}$ if and only if $\Gamma=\partial G$ has the following geometric property ([J]; see also $[\mathrm{M} 2]$ and $[\mathrm{R} 1])$ :

$$
\inf _{w_{1}, w_{2} \in \Gamma} \sup _{w \in\left(w_{1}, w_{2}\right)} \frac{\left|w_{1}-w\right|+\left|w_{2}-w\right|}{\left|w_{1}-w_{2}\right|}>1 ;
$$

here $\left(w_{1}, w_{2}\right)$ stands for the smaller subarc of $\Gamma$ between $w_{1}$ and $w_{2}$.

In the following theorem, (b) is essentially due to Stegenga and Stephenson [S-S], and (c) and (d) are similar to their descriptions of $\mathcal{B}_{0}$ and VMOA. 
Theorem 4.1. Let $f \in \mathcal{B}, f(0)=0$ and $\|f\|_{\mathcal{B}}=1$. Then

(a) $f \in \tilde{\mathcal{B}}$ if and only if $\omega_{a}(z)=0$ for all $a>0$ and all $z \in \mathbb{D}$.

(b) $f \in B M O A$ if and only if $\omega_{a}(z)>c$ for some numbers $a, c>0$ and all $z \in \mathbb{D}$.

(c) $f \in \mathcal{B}_{0}$ if and only if $l_{a}(z) \rightarrow 0$ as $|z| \rightarrow 1$, for all $a>0$.

(d) $f \in V M O A$ if and only if $\omega_{a}(z) \rightarrow 1$ as $|z| \rightarrow 1$ for all $a>0$.

(e) $f \in \mathcal{J}$ if and only if $l_{a}(z)>c$ for some numbers $a>e \pi / 4, c>0$, and all $z \in \mathbb{D}$.

Proof. (a) That $f \in \tilde{\mathcal{B}}$ implies $\omega_{a}(z)=0$ for all $z$ is [R2, (2.5)]. Next assume that $f \notin \tilde{\mathcal{B}}$. Then $f$ has radial limits on a set of positive measure and there is a constant $c$ so that $f$ is bounded by $c$ on a set of radii of positive measure. It follows that $\omega_{c}(0) \neq 0$.

(b) By Lemma 2.1 we have $G_{a} \subset \Omega_{a+K_{1}}$ so that

$$
\omega_{G_{a}}(\mathbb{T}) \leq \tilde{\omega}_{a+K_{1}}(z) .
$$

As $G_{a}$ is a chord-arc domain (with norm only depending on the angle $\beta<\pi / 2$ ) we have $\omega_{a}(z) \leq 1 / c \omega_{G_{a}}(\mathbb{T})^{c}$ for some universal $c$, so that (b) is equivalent to [S-S, Theorem A (ii)]. For later use we record that the proof of Theorem 2.2 in [S-S] shows

$$
\omega_{a}(z) \geq 1-A \exp \left(-\frac{B}{\|f\|_{*}} a\right)
$$

where $A$ and $B$ are universal constants.

(c) If $l_{a}(z) \rightarrow 0$ as $|z| \rightarrow 1$, for all $a>0$, then $f \in \mathcal{B}_{0}$ follows easily from [S-S, Theorem 1]: Indeed, Stegenga and Stephenson define

$$
r_{f}(z)=\sup \left\{a: \overline{\Omega_{a}(f, z)} \subset \mathbb{D}\right\}
$$

and show $f \in \mathcal{B}_{0}$ if and only if $r_{f}(z) \rightarrow 0$ as $|z| \rightarrow 1$. Since $l_{a}(z) \leq 1 / 2$ implies $\partial \Omega_{a} \cap \mathbb{T} \neq \emptyset$, we have

$$
r_{f}(z) \leq \sup \left\{a: l_{a}(z) \geq \frac{1}{2}\right\} \rightarrow 0 \quad \text { as }|z| \rightarrow 1,
$$

thus $f \in \mathcal{B}_{0}$.

Now let $f \in \mathcal{B}_{0}$. Fix $\zeta_{0} \in \mathbb{D}$ and define $T, \phi$ and $g=f \circ T \circ \phi$ as in the proof of Lemma 2.3, so that again $\|g\|_{\mathcal{B}} \leq \mu=\mu\left(\rho\left(\left|\zeta_{0}\right|\right)\right) \rightarrow 0$ as $\left|\zeta_{0}\right| \rightarrow 1$. If $a>\sqrt{\mu}$, then

$$
\left|I_{n}(g, 0, a)\right| \leq K_{2} \exp \left(-\frac{a}{\sqrt{\mu}}\right)
$$

by (2.4), and $l_{a}(z) \rightarrow 0$ (as $|z| \rightarrow 1$, for each $a$ ) follows.

(d) Let us first assume that $\omega_{a}(z) \rightarrow 1$ as $|z| \rightarrow 1$ for all $a>0$. We already know $f \in$ BMOA by (b). Let $z \in \mathbb{D}$ and $I(z) \subset \mathbb{T}$ be the corresponding arc (we fix $\beta$ ). By (4.1) and (c) we have $f \in \mathcal{B}_{0}$. Thus

$$
\frac{1}{|I(z)|} \int_{I(z)} f(x)|d x|-f(z) \rightarrow 0 \quad \text { as } \quad|z| \rightarrow 1
$$


By assumption, for every $a>0$ and $\varepsilon>0$ there is $\delta>0$ so that

$$
|\{x \in I(z):|f(x)-f(z)|>a\}|<\varepsilon|I(z)| \text { for } \quad|z|>1-\delta .
$$

Together with (4.5) this easily implies

$$
\frac{1}{|I(z)|} \int_{I(z)}|f(x)-f(z)||d x| \rightarrow 0 \quad \text { as } \quad|z| \rightarrow 1 .
$$

We have shown $f \in$ VMOA.

Conversely, let $f \in$ VMOA. Fix $\zeta_{0} \in \mathbb{D}$ and define $T, \phi$ and $g=f \circ T \circ \phi$ as in the proof of Lemma 2.3. It is not hard to show that $g$ is in BMOA with $\|g\|_{*} \rightarrow 0$ as $\left|\zeta_{0}\right| \rightarrow 1$. Now (4.4) easily implies $\omega_{a}(z) \rightarrow 1$ as $|z| \rightarrow 1$ for all $a>0$.

(e) Let $f \in \mathcal{J}$. As everything is Möbius invariant, all we have to show is $l_{a}(0)>$ $c$ for some constants $a$ and $c$ depending only on the constant $\varepsilon$ of (4.2). The computation in [J, section 4, Lemma 3] shows

$$
\int_{0}^{2 \pi}\left|f\left(r e^{i t}\right)-f(0)\right|^{2} d t \geq C \varepsilon^{2} \log \frac{1}{1-r}
$$

for $1 / 2 \leq r<1$ and some universal $C$. We conclude that for all $a>e / 2$ and some $r=r(a, \varepsilon)$ (not depending on $f$ )

$$
\partial \Omega_{a}(f, 0) \cap\{|z|=r\} \neq \emptyset .
$$

As $G_{a-K_{1}} \subset \Omega_{a}$ by Lemma 2.1, we also have

$$
\partial G_{a-K_{1}}(f, 0) \cap\{|z|=r\} \neq \emptyset
$$

if $a-K_{1}>e / 2$. This shows that $l_{a-K_{1}}(0) \geq c(1-r)$ (where $c$ depends only on $\beta$ and is thus fixed.)

To show the converse, set $\beta=\pi / 2$ and fix $z_{0} \in G$. Form the domain $G_{a}=$ $G_{a}\left(f, z_{0}\right)$ and note that $|f(z)| \geq a-e \pi / 4>0$ on $\partial G_{a} \cap \mathbb{T}$, since the constant $K_{1}$ in Lemma 2.1 is $e \pi / 4$ for $\beta=\pi / 2$ by [P, Theorem 4.2]. As $l_{a}\left(z_{0}\right)>c$, the hyperbolic distance of $\partial G_{a}$ to $z_{0}$ is bounded above by some constant $r$ (depending only on $c$ and $a$ ). Thus $\sup _{\rho\left(z, z_{0}\right)<r}\left(1-|z|^{2}\right)\left|f^{\prime}(z)\right|$ is bounded away from 0 , and it is easy to see that this implies the theorem.

As an application of (e), we mention the following: The class of quasicircles satisfying (4.3) is invariant under bilipschitz maps of the plane. This answers a question of M. Vuorinen (oral communication) (see $[\mathrm{V}-\mathrm{V}-\mathrm{W}]$ for related results). We will sketch an argument that mimics the proof of Bishop and Jones [B-J] that the class of simply connected domains $G$ with $\log g^{\prime} \in B M O A$ ( $g$ is the conformal map of $\mathbb{D}$ to $G$ ) is bilipschitz invariant; see also [A-Z].

First, it is clear that the class $\mathcal{C}$ of quasicircles described below is bilipschitz invariant: Say that $\Gamma \in \mathcal{C}$ if for every $C>1$ there is $\varepsilon>0$ so that the following holds for all points $a, b \in \Gamma$ and every curve $\gamma \subset \bar{G}=\overline{\operatorname{Int}(\Gamma)}$ with endpoints $a$ and $b$ : If length $(\gamma) \leq C|a-b|$, then there is a component $\tilde{\gamma}$ of $\gamma \cap G$ with length $(\tilde{\gamma}) \geq \varepsilon|a-b|$. Roughly speaking, $\Gamma \in \mathcal{C}$ if no subarc of $\Gamma$ can be approximated well by a rectifiable curve in $\bar{G}$. 
Next, observe that $\mathcal{C}$ is just the class of curves satisfying (4.3). A normal families argument shows that (4.3) implies membership in $\mathcal{C}$. Conversely, if $\Gamma \in \mathcal{C}$, consider a conformal map $g: \mathbb{D} \rightarrow G$ and set $f=\log g^{\prime}$. If $\Gamma$ does not satisfy (4.3), then $f \notin \mathcal{J}$, as mentioned above. Then (e) of Theorem 4.1 shows that there is $a>e \pi / 4$ and a sequence $z_{n}$ in $\mathbb{D}$ so that $l_{a}\left(z_{n}\right) \rightarrow 0$ as $n \rightarrow \infty$. With $\beta=\pi / 2$, consider the domain $G_{a}=G_{a}\left(f, z_{n}\right)$ and the $\operatorname{arc} I\left(z_{n}\right) \subset \mathbb{T}$. Let $\sigma_{n}$ be the largest subarc of $\partial G_{a}$ that has both endpoints on $I_{n}$ and satisfies $\overline{\sigma_{n}} \cap \mathbb{T} \subset I_{n}$. As $\exp (-e \pi / 4) \leq\left|f^{\prime}(z) / f^{\prime}\left(z_{n}\right)\right| \leq$ $\exp (e \pi / 4)$ on $\partial G_{a}$, integration of $\left|f^{\prime}\right|$ and standard estimates show that the curves $\gamma_{n}=f\left(\sigma_{n}\right)$ satisfy length $\left(\gamma_{n}\right) \leq K\left(1-\left|z_{n}\right|\right)\left|f^{\prime}\left(z_{n}\right)\right| \leq K^{\prime}\left|a_{n}-b_{n}\right|$, where $a_{n}$ and $b_{n}$ are the endpoints of $\gamma_{n}$. But every component $\tilde{\gamma}_{n}$ of $\gamma_{n} \cap G$ corresponds to a (circular) arc on $\partial G_{a}$. As $l_{a}\left(z_{n}\right) \rightarrow 0$ we obtain length $\left(\tilde{\gamma}_{n}\right)=o\left(\operatorname{length}\left(\gamma_{n}\right)\right)$ as $n \rightarrow \infty$, contradicting $\Gamma \in \mathcal{C}$.

\section{REFERENCES}

[A-C-P] J.Anderson,J.Clunie,C.Pommerenke, On Bloch functions and normal functions, J.Reine Ang.Math. 270 (1974), 12-37. MR 50:13536

[A-P] J.Anderson,L.Pitt, The boundary behaviour of Bloch functions and univalent functions, Mich.Math.J 35 (1988), 313-320. MR 89m:30067

[A-Z] K.Astala,M.Zinsmeister, Teichmüller spaces and BMOA, Math.Ann. 289 (1991), 613625. MR 92k:30031

[B] C.Bishop, Bounded functions in the little Bloch space, Pacific J.Math. 142 (1990), 209225. MR 91b:30101

[B-J] C.Bishop, P.Jones, Harmonic measure, $L^{2}$-estimates and the Schwarzian derivative, J. Anal. Math. 62 (1994), 77-113. MR 95f:30034

[C-C-P1] J.Carmona, J.Cufi, C.Pommerenke, On the angular limits of Bloch functions, Publ. Mat. 32 (1988), 191-198. MR 89m:30071

[C-C-P2] J.Carmona, J.Cufi, C.Pommerenke, On the angular boundedness of Bloch functions, Revista Mat. Iberoamericana 5 (1989), 125-137. MR 91m:30043

[G] J.Garnett, Bounded analytic functions, Academic Press, 1981. MR 83g:30037

[GH-P] D.Gnuschke-Hauschild,C.Pommerenke, On Bloch functions and gap series, J.Reine Ang. Math. 367 (1986), 172-196. MR 88a:30072

[H] G.Hungerford, Boundaries of smooth sets and singular sets of Blaschke products in the little Bloch space, Thesis, Pasadena (1988).

[J] P.Jones, Square functions, Cauchy integrals, analytic capacity and harmonic measure, Harmonic Analysis and Partial Differential Equations (El Escorial, 1987), Lecture Notes in Mathematics, vol. 1384, Springer, 1989, pp. 24-68. MR 91b:42032

[ML] G.MacLane, Asymptotic values of holomorphic functions, Rice University Studies 49 (1963). MR 26:6419

[M1] N.G.Makarov, Conformal mapping and Hausdorff measures, Ark.Mat. 25 (1987), 41-89. MR 89g:30044

[M2] N.G.Makarov, Probability methods in the theory of conformal mappings, Leningrad Math. J. 1 (1990), 1-56. MR 90k:30008

[P] Ch. Pommerenke, Boundary behaviour of conformal maps, Springer, 1992. MR 95b: 30008

[R1] S.Rohde, On conformal welding and quasicircles, Michigan Math. J. 38 (1991), 111-116. MR 92f:30011

[R2] S.Rohde, The boundary behavior of Bloch functions, J.London Math.Soc. 48 (1993), 488-499. MR 94k:30083

[S-S] D.Stegenga, K.Stephenson, A geometric characterization of analytic functions with bounded mean oscillation, J.London Math.Soc.(2) 24 (1981), 243-254. MR 82m:30036

[V-V-W] J.Väisälä, M.Vuorinen, H.Wallin, Thick sets and quasiregular maps, preprint.

Department of Mathematics, University of Michigan, Ann Arbor, Michigan 48109

Fachbereich Mathematik, TU Berlin, 10623 Berlin, Germany

E-mail address: rohde@math.tu-berlin.de 